\title{
Diabatization by localization in the framework of configuration interaction based on floating occupation molecular orbitals (FOMO-CI).
}

\author{
Davide Accomasso * Prof. Maurizio Persico ${ }^{\dagger *}$ \\ Prof. Dr. Giovanni Granucci *
}

This work is dedicated to Jean-Paul Malrieu on the occasion of his 80th birthday.

\section{Abstract}

We present a diabatization method of general applicability, based on the localization of molecular orbitals on user specified groups of atoms. The method yields orthogonal molecular orbitals similar to the canonical ones for the isolated atom groups, that are the basis to build reference spin-adapted configurations representing localized or charge transfer excitations. An orthogonal transformation from the adiabatic to the quasi-diabatic basis is defined by requiring maximum overlap with the diabatic references. We present the diabatization algorithm as implemented in the framework of semiempirical configuration interaction based on floating occupation molecular orbitals (FOMO-CI), but the same transformation can also be applied to ab initio wavefunctions, obtained for instance with state-average CASSCF. The diabatic representation so obtained and the associated hamiltonian matrix are particularly suited to assess quantitatively the interactions that account for charge and energy transfer transitions, and to analyze the results of nonadiabatic dynamics simulations involving such phenomena.

\section{Introduction}

The diabatic representation [1-6] serves various important purposes in the theory and calculation of excited state dynamics. First, it is a convenient tool to characterize the nature of the electronic states and to analyze the dynamics [7-9]. Second, it minimizes the derivative couplings, thus being the preferred choice in surface crossing situations where such couplings become very large or

\footnotetext{
*Dipartimento di Chimica e Chimica Industriale, Università di Pisa, v. G. Moruzzi 13, I-56124 Pisa, Italy

${ }^{\dagger}$ corresponding author
} 
even diverge [10-14] (a set of states whereby the derivative couplings are small, rather than identically vanishing, is more correctly qualified as "quasi-diabatic", but we shall use the word diabatic with just this meaning). Probably most important, the hamitonian matrix in the diabatic basis carries all the information about the adiabatic potential energy surfaces (PES), that are its eigenvalues, and also the essential information about the couplings, while being much more easily represented in analytic form for interpolation and fitting [15] or modelling $[16,17]$ purposes.

The diabatic basis can be determined by variously defined transformations of previously computed adiabatic states $[3,10-14,18,19]$, or by a direct approach, i.e. by identifying a set of (most often non orthogonal) wavefunctions that are smoothly dependent on the nuclear coordinates [20-26]. In the latter case, the construction of the hamiltonian and overlap matrices in the diabatic basis allows to obtain the adiabatic states. The definition of the diabatic representation is most intuitive and their use is most appealing, when dealing with excitation or charge transfer processes in multi-chromophoric systems [4,21-26]. In such cases, the direct approach can be a very efficient alternative to traditional electronic structure methods, because the diabatic wavefunctions can be obtained as products of factors separately optimized for each subsystem [21-26]. However, the accuracy of the results depends on the correct identification of the diabatic basis, which must be assessed case by case [8].

In this paper we present a method to identify a diabatizing transformation of a set of adiabatic states for a system that can be clearly separated into subsystems (the "monomers"), such that each diabatic state can be characterized by the localization of charge and excitation. The method consists of two steps: first, the relevant molecular orbitals (MO) are rotated to produce MOs localized on the monomers (LMO). Next, diabatic reference states are built on the LMO basis and the adiabatic states are transformed so as to achieve maximum overlap with the references. The aim is to be able to analyze adiabatic or time-dependent electronic wavefunctions, obtained for instance in excited state dynamics simulations [9], and to assess the interactions that determine exciton delocalization, charge transfer, singlet fission and other phenomena occurring in multi-chromophoric systems. In the first place the method was implemented in the framework of semiempirical configuration interaction based on floating occupation molecular orbitals (FOMO-CI) $[5,10]$. However, it can be applied without changes to ab initio wavefunctions, obtained for instance by state-average CASSCF or ab initio FOMO-CI $[27,28]$. Section 2 presents the theory and section 3 contains some test applications. 


\section{Theory}

\subsection{Localization of molecular orbitals on pre-defined sub- systems by singular value decomposition}

Orbital localization methods have been devised either to improve computational efficiency or to quantify the role of chemical groups in the electronic wavefunctions (see [6], section 6.6). We are interested in the latter class of localization methods $[29,30]$ and in particular we require the following properties: (a) the subsets of atoms defining the monomers can be chosen on physical grounds; (b) only a subset of active orbitals undergo localization; (c) the LMOs are orthogonal; (d) the LMOs resemble the isolated monomer canonical orbitals.

We start from a subset of orthonormal MOs $\left\{\varphi_{i} \mid i=1,2, \ldots, n\right\}$ defined as linear combinations of normalized atomic orbital (AO) basis functions $\left\{\chi_{\mu} \mid \mu=\right.$ $1,2, \ldots, m\}$ with expansion coefficients $\left\{C_{\mu i}\right\}$ :

$$
\varphi_{i}=\sum_{\mu=1}^{m} \chi_{\mu} C_{\mu i} \quad i=1,2, \ldots, n .
$$

We partition our molecular system into $M$ groups of atoms, the monomers. Each monomer $X$ is associated with $m_{X}$ basis functions centered on the atoms belonging to it. Thus, we can rewrite eq. 1 as follows:

$$
\varphi_{i}=\sum_{X=1}^{M} \sum_{\mu \in X}^{m_{X}} \chi_{\mu} C_{\mu i} \quad i=1,2, \ldots, n
$$

where for each monomer $X$ the expansion coefficients $\left\{C_{\mu i}\left|\mu=1,2, \ldots, m_{X}\right| i=\right.$ $1,2, \ldots, n\}$ form an $m_{X} \times n$ matrix $\mathbf{C}_{X}$, corresponding to a block of the $m \times n$ matrix of $\mathrm{MO}$ coefficients $\mathbf{C}$. To identify a unitary transformation of the original MOs such that each new orbital is maximally localized on one monomer, we resort to singular value decomposition (SVD), which was previously used in a related context [31]. For each monomer $X$, we perform the SVD of $\mathbf{C}_{X}$ :

$$
\mathbf{C}_{X}=\mathbf{U}_{X} \boldsymbol{\Sigma}_{X} \mathbf{V}_{X}^{T}
$$

where $\mathbf{U}_{X}$ and $\mathbf{V}_{X}$ are orthogonal matrices of dimension $m_{X}$ and $n$ respectively, whose columns $\mathbf{U}_{X, i}, \mathbf{V}_{X, i}$ are called the left and right singular vectors of $\mathbf{C}_{X}$, and $\boldsymbol{\Sigma}_{X}$ is an $m_{X} \times n$ diagonal matrix, whose diagonal entries, $\sigma_{X, 1}, \sigma_{X, 2}, \ldots \sigma_{X, n}$, non-negative by definition, are called the singular values of $\mathbf{C}_{X}$ (for more information about the SVD, see Section S1). If we rotate the original MOs according to $\mathbf{V}_{X}$, we obtain:

$$
\bar{\varphi}_{i}=\sum_{j=1}^{n} \varphi_{j} V_{X, j i}=\sum_{\mu \in X}^{m_{X}} \chi_{\mu}\left(U_{X, \mu i} \sigma_{X, i}\right)+\sum_{Y(\neq X)}^{M} \sum_{\mu \in Y}^{m_{Y}} \chi_{\mu}\left(\sum_{j=1}^{n} C_{Y, \mu j} V_{X, j i}\right)
$$

where for the new MO coefficients of the AOs on monomer $X$ we have exploited eq. 3 , in particular the relation $\mathbf{C}_{X} \mathbf{V}_{X, i}=\mathbf{U}_{X, i} \sigma_{X, i}$. Adding up the squares 
of the expansion coefficients of $\bar{\varphi}_{i}$ from the AOs on monomer $X$, we get:

$$
\sum_{\mu \in X}^{m_{X}}\left(U_{X, \mu i} \sigma_{X, i}\right)^{2}=\sigma_{X, i}^{2} \sum_{\mu \in X}^{m_{X}} U_{X, \mu i}^{2}=\sigma_{X, i}^{2} .
$$

Therefore, for the transformed MOs we can define the indices of localization on monomer $X$ in this way:

$$
\lambda_{X, i}=\frac{\sum_{\mu \in X}^{m_{X}} \bar{C}_{\mu i}^{2}}{\sum_{\nu=1}^{m} \bar{C}_{\nu i}^{2}}=\frac{\sigma_{X, i}^{2}}{\sum_{\nu=1}^{m} \bar{C}_{\nu i}^{2}}
$$

where $\left\{\bar{C}_{\nu i}|\nu=1,2, \ldots, m| i=1,2, \ldots, n\right\}$ are the expansion coefficients of the new $\operatorname{MOs}\left\{\bar{\varphi}_{i}\right\}$ (eq. 4). Since $0 \leq \lambda_{X, i} \leq 1$, the closer to $1 \lambda_{X, i}$ is, the more $\bar{\varphi}_{i}$ is localized on monomer $X$. Moreover, $1-\lambda_{X, i}$ indicates how much $\bar{\varphi}_{i}$ is delocalized on the remainder of the system.

Subsequently, among the $n \cdot M$ right singular vectors obtained from the SVD of $\left\{\mathbf{C}_{X} \mid X=1,2, \ldots, M\right\}$, we select the ones associated with the $n$ largest $\lambda_{X, i}$. Since these $n$ vectors may result from SVDs of different submatrices of $\mathbf{C}$, they might not be orthogonal to each other. Therefore, we Löwdin orthogonalize [32] them and use the column vectors obtained to define an $n \times n$ orthogonal matrix $\mathbf{V}$ for the rotation of the original MOs:

$$
\overline{\overline{\varphi_{i}}}=\sum_{j=1}^{n} \varphi_{j} V_{j i} \quad i=1,2, \ldots, n
$$

If we work with orthogonal AO basis functions, as in the case of NDO (neglect of differential overlap) semiempirical methods [33-36], the denominator of eq. 6 is simply 1 . The same is true with ab initio methods, if the MO coefficients $\mathbf{C}$ refer to a Löwdin orthogonalized basis set. Hence, the squares of the singular values of $\mathbf{C}_{X}$ represent our localization indices, i.e. $\lambda_{X, i}=\sigma_{X, i}^{2}$. In this context, if the system is divided into two monomers, $A$ and $B$, the orthogonal matrix $\mathrm{V}$ of eq. 7 can be obtained by simply performing the SVD of one submatrix of $\mathbf{C}$, either $\mathbf{C}_{A}$ or $\mathbf{C}_{B}$. In fact, since the MOs, as well as the AO basis functions, form an orthonormal set, we have:

$$
\left\langle\varphi_{i} \mid \varphi_{j}\right\rangle=\delta_{i j}=\sum_{\mu \in A}^{m_{A}} C_{\mu i} C_{\mu j}+\sum_{\nu \in B}^{m_{B}} C_{\nu i} C_{\nu j} \quad i, j=1,2, \ldots, n
$$

which in matrix form is $\mathbf{C}_{A}^{T} \mathbf{C}_{A}+\mathbf{C}_{B}^{T} \mathbf{C}_{B}=\mathbf{1}$. Therefore, $\mathbf{C}_{A}^{T} \mathbf{C}_{A}$ and $\mathbf{C}_{B}^{T} \mathbf{C}_{B}=$ $\mathbf{1}-\mathbf{C}_{A}^{T} \mathbf{C}_{A}$ have the same eigenvectors which correspond to the right singular vectors of both $\mathbf{C}_{A}$ and $\mathbf{C}_{B}$. In other words, if $\mathbf{U}_{A} \boldsymbol{\Sigma}_{A} \mathbf{V}_{A}^{T}$ and $\mathbf{U}_{B} \boldsymbol{\Sigma}_{B} \mathbf{V}_{B}^{T}$ are the SVD of $\mathbf{C}_{A}$ and $\mathbf{C}_{B}$, respectively, it turns out that $\mathbf{V}_{A}=\mathbf{V}_{B}$ and the singular values are such that $\sigma_{A, i}^{2}=1-\sigma_{B, i}^{2}$. 


\subsection{Transformation of localized orbitals by constrained SCF with floating occupation}

Provided that the choice of the $n$-dimensional MO space is made properly, the procedure described in the previous section gives well localized orbitals. However, since in the SVD we have no control on the mixing of the localized orbitals in each monomer, generally the new MOs obtained do not resemble the SCF orbitals of the isolated monomers. Therefore, one may want to further rotate the orbitals localized on each fragment, to make them more similar to the canonical or natural orbitals of the isolated fragment. This can be achieved by blockdiagonalization of the Fock matrix or of the density matrix. In particular, if the $\mathrm{MO}$ localization is used in conjunction with the FOMO-CI method, we perform a constrained SCF calculation where at each iteration the Fock matrix is diagonalized within each block of orbitals localized on the same monomer. In the SCF procedure we define the 1-electron density matrix $\mathbf{D}$ by introducing fractional occupation numbers $\left\{O_{i} \mid i=1,2, \ldots, n\right\}$ for the active orbitals, the same that undergo the localizing transformation:

$$
D_{\mu \nu}=\sum_{i=1}^{n} O_{i} C_{\mu i} C_{\nu i} \quad \mu, \nu=1,2, \ldots, m .
$$

The fractional occupation numbers fall in the interval $[0,2]$ and may be either arbitrarily fixed or floating. In the latter case, we partition the total number of electrons $N_{e l}$ among the $M$ monomers, so as to assign them predefined charges, and, at each SCF iteration, we determine the occupation numbers according to the diagonal elements of the Fock matrix (orbital energies) $\left\{\epsilon_{X, i}\left|i=1,2, \ldots, n_{X}\right| X=1,2, \ldots, M\right\}$, with $n_{X}$ indicating the number of MOs localized on monomer $X$ :

$$
O_{X, i}=\frac{\sqrt{2}}{\sqrt{\pi} w} \int_{-\infty}^{\epsilon_{F}^{X}} e^{-\frac{\left(\epsilon-\epsilon_{X, i}\right)^{2}}{2 w}} d \epsilon
$$

where $\left\{\epsilon_{F}^{X}\right\}$ are the Fermi levels, one for each monomer, and $w$ is the orbital energy width, an arbitrarily chosen parameter which determines the spread of electronic population below and above the Fermi levels. The latter are set by imposing that the sum of the occupation numbers is equal to the total number of electrons, within each monomer, i.e. $N_{e l}^{X}=\sum_{i=1}^{n_{X}} O_{X, i}$, where $N_{e l}^{X}$ is the number of electrons assigned to monomer $X$. In this way the localized MOs are computed for monomers with predefined charges (usually neutral). Note that FOMO-CI is not a size-consistent method, even in its complete active space version (see next section), because the Fermi level obtained in a calculation with not interacting but not identical monomers differs from the Fermi levels of separate calculations for the individual monomers, therefore the SCF density matrix and the MOs also differ.

Once the SCF convergence is reached, the final MOs minimize the SCF energy and acquire high resemblance with the canonical SCF orbitals of the isolated monomers, still preserving the degree of localization of the starting MOs. 


\subsection{Construction of diabatic states}

When a CI calculation is based on localized MOs, a simple inspection of the CI coefficients can suffice to characterize the computed adiabatic states. However, such analysis can be tedious and prone to accidental mistakes, especially with large CI expansions. Moreover, a quantitative evaluation of the energies of states of different nature (localized excitations, charge transfer etc) and of the interactions between them, requires the unambiguous identification of a diabatic representation. The MOs obtained from the localization procedure described in the previous sections can be exploited to construct diabatic electronic states. In particular, one can define the diabatic wavefunctions as linear combinations of previously obtained adiabatic states and determine the unitary transformation from the adiabatic basis to the diabatic one by making use of diabatic references based on the localized MOs, as we did in previous work [18]. In our approach, we define the rotation matrix so that the sum of the overlaps between the diabatic wavefunctions and the reference states is maximized:

$$
\sum_{j=1}^{N}\left\langle\Phi_{j}^{(D)} \mid R_{j}\right\rangle=\max
$$

where $\left\{\Phi_{j}^{(D)}\right\}$ and $\left\{R_{j}\right\}$ are the diabatic states and the references, respectively. This maximization is achieved by, first, projecting $\left\{R_{j}\right\}$ onto the space spanned by the adiabatic states and, then, applying a Löwdin orthogonalization [32] on the resulting matrix of projections (see Section S2):

$$
\begin{gathered}
\Phi_{j}^{(D)}=\sum_{k=1}^{N} \Phi_{k}^{(A)} T_{k j} \quad j=1,2, \ldots, N \\
T_{k j}=\sum_{l=1}^{N}\left\langle\Phi_{k}^{(A)} \mid R_{l}\right\rangle\left(S^{-1 / 2}\right)_{l j}
\end{gathered}
$$

where $\mathbf{T}$ is the rotation matrix of the adiabatic states $\left\{\Phi_{j}^{(A)}\right\}$ to obtain the diabatic ones, and $\mathbf{S}$ is the overlap matrix between the projections of the reference states onto the adiabatic basis, i.e. $S_{l j}=\left\langle\Phi_{l}^{(D)} \mid \Phi_{j}^{(D)}\right\rangle$, with $\bar{\Phi}_{l}^{(D)}=$ $\sum_{k=1}^{N} \Phi_{k}^{(A)}\left\langle\Phi_{k}^{(A)} \mid R_{l}\right\rangle$.

In our case, the adiabatic states are computed with a variant of the FOMOCI method, in which the configuration interaction is of the complete active space (CAS) type $[10,37]$ This can be viewed as a convenient substitute for the complete active space self-consistent field (CASSCF) method. In particular, in the FOMO-CASCI procedure the MOs are partitioned into inactive, active and virtual. In the SCF the inactive orbitals are doubly occupied, the virtual MOs are unoccupied and the active ones have fractional occupations, either fixed or floating. In the latter case, the occupation numbers are defined as in eq. 10, with a unique Fermi level $\left(\epsilon_{F}^{X}=\epsilon_{F}\right)$. Then, the adiabatic electronic states are obtained through a CASCI calculation, i.e. a full CI within the active 
orbital space. When we want to construct diabatic states, on top of the SCF we localize the active MOs as described in Sections 3.1 and 3.2. Subsequently, we perform the CASCI calculation by making use of Slater determinants built on the localized active MOs. Since in the CASCI method the adiabatic electronic states are linear combinations of all the excitations within the active orbitals, a unitary transformation of the active MOs does not alterate the expectation value of any observable property, including the energy.

Regarding the reference states, they are simple wavefunctions with a well defined character (ionic or neutral, localized or charge transfer excitation, etc.). In particular, each reference state is made of one or few configurations built on the localized MOs, namely the configurations which are supposed to be dominant in the corresponding diabatic state thoughout the coordinate space of interest. Moreover, since our localized MOs resemble the canonical SCF orbitals of the isolated monomers, to construct the references we can exploit the information obtained from the calculations on the single monomers. For instance, suppose we are studying a pair of interacting identical chromophores, $A$ and $B$, with $A=B$. From the calculations on one isolated monomer we get that the ground state $S_{0}$ is dominated by the closed-shell Hartree-Fock wavefunction, while the first singlet excited state $S_{1}$ is mainly described as transitions of one electron from the HOMO to the LUMO orbital of each monomer. Thus, if we want to construct a diabatic state the character of which is mainly a single excitation localized on one monomer, say $A$, i.e. $\Phi^{(D)}=S_{1}(A) S_{0}(B)$, we may use as reference state the configuration $\frac{1}{\sqrt{2}}\left(\left|\cdots h_{A} \overline{l_{A}} h_{B} \overline{h_{B}}\right|-\left|\cdots \overline{h_{A}} l_{A} h_{B} \overline{h_{B}}\right|\right)$, where $h_{X}$ and $l_{X}$, with $X=A$ or $B$, are the localized MOs of the dimer which mainly resemble the HOMO and LUMO of monomer $X$.

\section{Applications}

In most applications of the FOMO-CI method, the semiempirical Hamiltonian is chosen and reparameterized in order to reproduce high quality ab initio and experimental data concerning the specific molecular system and processes under study (see for instance ref. [38]). The selection of the CI space and of the energy width parameter $w$ are also involved in the optimization procedure. In the three examples here discussed, we did not optimize the semiempirical parameters and we limited ourselves to the choice of standard Hamiltonians that yielded reasonable results. Actually the agreement with experimental data is quite good for the first case study we present, 1,3-diphenylisobenzofuran.

\subsection{Singlet fission in a dimer of 1,3-diphenylisobenzofuran}

Singlet fission is a photophysical process where a spin singlet excited state is converted into two lower energy spin triplet excitons [39-42]:

$$
S^{*} \rightarrow T+T .
$$


The initial singlet excited state $S^{*}$ is typically generated by absorption of one photon and may be delocalized over more chromophores as a result of exciton coupling between chromophores in their $S_{1}$ state. The two triplet excitons, each located on one chromophore, are generally $T_{1}$ states. They can combine to form an overall singlet state, hereafter indicated as ${ }^{1} T T$, making the singlet fission process spin-allowed and hence potentially fast (namely it can occur on a picosecond or even sub-picosecond time scale $[43,44]$ ).

Since singlet fission is a downconversion process of high-energy photons into lower-energy excitons, it can be exploited in photovoltaics to improve the solar energy conversion efficiency in the high-frequency part of the solar spectrum [45], by overcoming the so-called Shockley-Queisser limit [46].

The singlet fission quantum yield is highly sensitive to the electronic couplings between the diabatic states involved, i.e. the localized excitations, the charge transfer states and the ${ }^{1} T T$ state. The couplings depend on the mutual placement of the chromophores, so detailed searches for the optimal relative arrangements are an important strategy for the development of singlet fission materials. Our diabatization procedure can be employed to perform such an investigation. In particular, it can be used to identify the optimal geometry for a pair of rigid molecules, as in work [47], or to select the most favourable dimer among the different pairs of neighbouring molecules within a crystal structure $[48,49]$.

Moreover, in a simulation of the excited state dynamics, as in $[9,49]$, our diabatization method allows to analyze the relative importance of the different mechanisms so far identified to explain singlet fission: (i) the direct mechanism, which consists in the population of the ${ }^{1} T T$ state directly from the initial excited state $S^{*}$; (ii) the mediated one, involving higher-energy (virtual) states, generally identified to be of charge transfer character, which are hardly populated but assist the formation of the ${ }^{1} T T$ state through the so-called superexchange effect; (iii) the two step mechanism, where low-lying intermediate states, possibly of charge transfer character, are actually populated before the generation of ${ }^{1} T T[39,40,50]$.
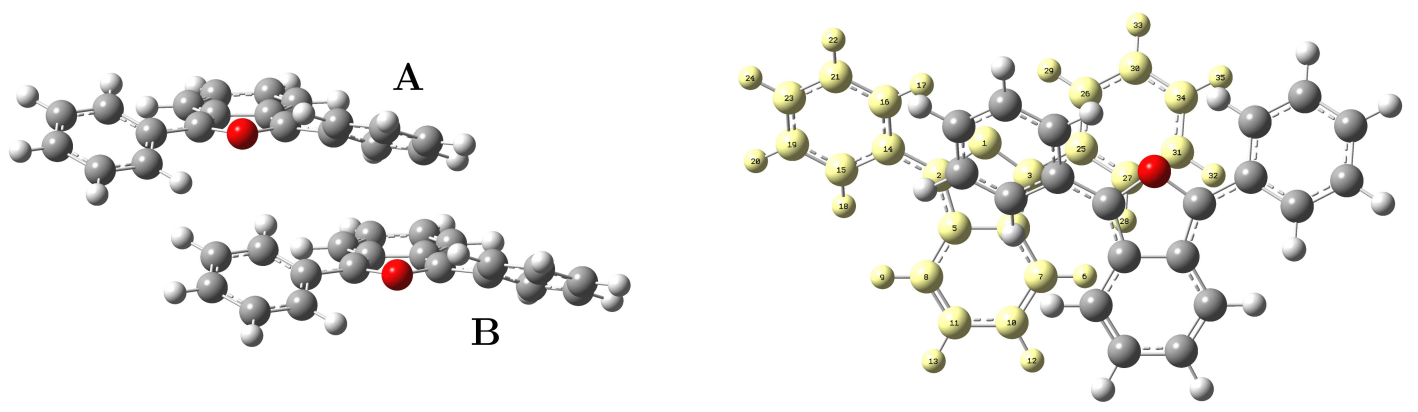

Figure 1: Slip-stacked dimer model of DPBF. Two different views are shown. $A$ and $B$ are used to label the two monomers. 
Here, as an example, we present the calculation of the electronic couplings between the low-lying singlet diabatic states for a dimer of 1,3-diphenylisobenzofuran (DPBF), the first successful case of a chromophore specifically designed for singlet fission $[51,52]$.

We computed the adiabatic electronic states of the dimer using the AM1 [34] semiempirical Hamiltonian and an active space of 4 electrons in 6 MOs. Moreover, the floating occupation numbers were determined using a gaussian energy width of 0.1 a.u. At this level of theory, using an active space of 2 electrons in 3 orbitals, the excitation energies of the monomer are in good agreement with the best computed and spectroscopic data (see Table 1).

Among the different dimers that can be identified in the efficient crystalline form $\alpha$ of DPBF [53] (triplet quantum yield of $\sim 140 \%$, determined at room temperature [54]) we chose the dimer with a slip-stacked arrangement, which, according to theoretical considerations based on a simplified HOMO/LUMO model $[39,40,55,56]$, was suggested to be a favourable mutal disposition for singlet fission. Then, we built a model of dimer by disposing two molecules, each at the AM1/FOMO-CASCI $(2,3)$ optimized $\mathrm{S}_{0}$ geometry (point group $C_{2}$ ), so as to reproduce the selected arrangement (see Fig. 1). In the dimer, the distance between the planes of the two isobenzofuran moieties is approximately of $4 \AA$.

Table 1: Vertical and adiabatic excitation energies (in $\mathrm{eV}$ ) for the $\mathrm{S}_{1}, \mathrm{~S}_{2}, \mathrm{~T}_{1}$ and $\mathrm{T}_{2}$ states of DPBF computed with the FOMO-CASCI method, using the AM1 semiempirical Hamiltonian and an active space of 2 electrons in 3 orbitals. In the calculations, the point group $\mathrm{C}_{2}$ is used. The results are compared with the best computed and spectroscopic data.

\begin{tabular}{c|cc|cc|c}
\hline $\begin{array}{c}\text { State } \\
\text { (label) }\end{array}$ & AM1 & $\begin{array}{c}\text { Vertical } \\
\text { Ref. value }\end{array}$ & AM1 & $\begin{array}{c}\text { Adiabatic } \\
\text { Ref. value }\end{array}$ & Exc. nature $^{h}$ \\
\hline $\mathrm{S}_{1}\left(1^{1} \mathrm{~B}\right)$ & $3.07^{i}$ & $3.01^{a}, 3.22^{b}, 2.92^{c}$ & 2.88 & $2.76^{e}, 2.98^{b}, 2.70^{c}$ & $h \rightarrow l$ \\
$\mathrm{~S}_{2}\left(1^{1} \mathrm{~A}\right)$ & $3.79^{j}$ & $3.67^{a}, 4.14^{b}$ & - & - & $h \rightarrow l+1,(h \Rightarrow l)$ \\
$\mathrm{T}_{1}\left(1^{3} \mathrm{~B}\right)$ & 1.82 & $2.05^{b}, 1.4^{d}$ & 1.36 & $1.41^{f}, 1.48^{g}$ & $h \rightarrow l$ \\
$\mathrm{~T}_{2}\left(1^{3} \mathrm{~A}\right)$ & 3.51 & $3.53\left(2^{3} \mathrm{~B}\right)^{b}, 3.1^{d}$ & - & - & $h \rightarrow l+1$ \\
\hline
\end{tabular}

${ }^{a}$ Absortion spectrum in 3-methylpentane at $77 \mathrm{~K}$, band maximum [57].

${ }^{b}$ Computed with the RI-CC2/TZVPP method, using the point group $\mathrm{C}_{2}$ [57].

${ }^{c}$ Computed at the TDDFT level (B3LYP/6-31G ${ }^{* *}$ ) [58].

${ }^{d}$ Computed at the TDDFT level (B3LYP/SV (P)) [51].

${ }^{e}$ Fluorescence spectrum in cyclohexane, 0-0 transition [57].

$f$ Electron energy loss spectroscopy in a solid film [57].

${ }^{g}$ Determined by sensitization in benzene [59].

${ }^{h}$ According to the AM1, FOMO-CASCI $(2,3)$ calculations. Notation for the orbitals: $h$, HOMO; $l$, LUMO. The symbol $\Rightarrow$ indicates double excitation.

${ }^{i}$ At the AM1, FOMO-CASCI $(2,3)$ level, the oscillator strength is 0.685 , in quite good agreement with the the RI-CC2/TZVPP value of 0.52 reported in [57].

$j$ At the AM1, FOMO-CASCI $(2,3)$ level, the oscillator strength is 0.404 , which is far from the RI-CC2/TZVPP value of 0.076 reported in [57]. 
For the determination of the diabatic states, we employed eight reference states. They are the spin singlet configurations schematically illustrated in Fig. 2 (see also Section S3). The first six references are prototypes for diabatic states which are generally considered to be the most involved in singlet fission [39,40], that is the ground state, $S_{0}(A) S_{0}(B)$, the localized excitations, $S_{1}(A) S_{0}(B)$ and $S_{0}(A) S_{1}(B)$, the singlet combination of the two triplets, ${ }^{1} T T$, and the charge transfer states $A^{-} B^{+}$and $A^{+} B^{-}$, where $A$ and $B$ indicate the two monomers. Since in the isolated molecule the $S_{2}-S_{1}$ energy gap is quite small (see Table 1 ), we decided to add two more references as prototypes for diabatic states with higher-energy, optically weakly allowed [57], local excitation character, that is $S_{2}(A) S_{0}(B)$ and $S_{0}(A) S_{2}(B)$.

The electronic Hamiltonian matrix in the diabatic basis computed for the slip-stacked dimer model of DPBF is reported in Table 2. We notice that the direct couplings $\left\langle S_{1}(A) S_{0}(B)\left|\hat{\mathcal{H}}_{e l}\right|{ }^{1} T T\right\rangle$ and $\left\langle S_{0}(A) S_{1}(B)\left|\hat{\mathcal{H}}_{e l}\right|^{1} T T\right\rangle$ are very small (less than $0.10 \mathrm{meV}$ ), while the matrix elements which couple both the localized excitations, $S_{1}(A) S_{0}(B)$ and $S_{0}(A) S_{1}(B)$, and ${ }^{1} T T$ with the charge transfer states, $A^{-} B^{+}$and $A^{+} B^{-}$, are much larger. Moreover, large matrix elements couple $S_{1}(A) S_{0}(B)$ and $S_{0}(A) S_{1}(B)$ with the higher-energy local excitations $S_{2}(A) S_{0}(B)$ and $S_{0}(A) S_{2}(B)$, which in turn show moderate electronic couplings with the ${ }^{1} T T$ state. However, while large energy gaps separate the charge transfer states from the local excitations $S_{1}(A) S_{0}(B)$ and $S_{0}(A) S_{1}(B)$, the latter, as well as the ${ }^{1} T T$ state, are much closer in energy to the $S_{2}(A) S_{0}(B)$ and $S_{0}(A) S_{2}(B)$ states. Thus, starting from the optically excited $S_{1}(A) S_{0}(B)$ and $S_{0}(A) S_{1}(B)$ states, the interactions mediated by both the charge transfer

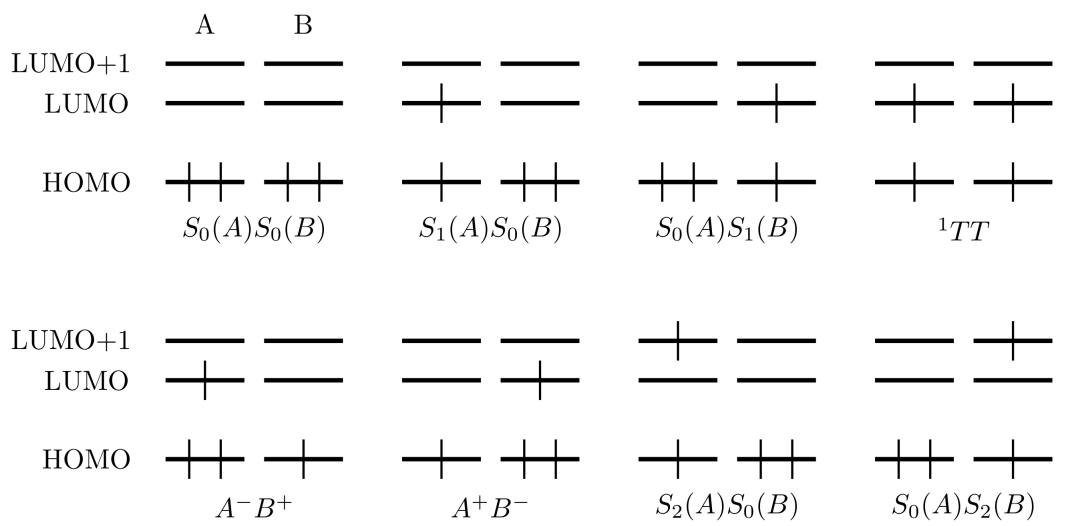

Figure 2: Schematic representation of the eight reference states used as prototypes for the construction of the diabatic states for the dimer model of DPBF. The horizontal bars indicate the localized MOs, while the vertical bars stand for electrons. $A$ and $B$ are used to label the two monomers. The references are indicated using the labels of the corresponding diabatic states. 
states and the higher-energy local excitations appear to play the fundamental role in the population of the ${ }^{1} T T$ state.

The importance of the mediated interactions can be quantitatively evaluated by making use of the effective Hamiltonian theory (see Section S4). In particular, the effective Hamiltonian matrix obtained by including the effect of $A^{-} B^{+}$, $A^{+} B^{-}, S_{2}(A) S_{0}(B)$ and $S_{0}(A) S_{2}(B)$, which define the outer space, on the electronic couplings between the model states $S_{1}(A) S_{0}(B), S_{0}(A) S_{1}(B)$ and ${ }^{1} T T$ is shown in Table 4. We see that $\left\langle S_{1}(A) S_{0}(B)\left|\hat{\mathcal{H}}_{\text {el }}^{\text {eff }}\right|{ }^{1} T T\right\rangle$ and $\left\langle S_{0}(A) S_{1}(B)\left|\hat{\mathcal{H}}_{\text {el }}^{e f f}\right|{ }^{1} T T\right\rangle$ are significantly larger than the corresponding matrix elements of $\hat{\mathcal{H}}_{e l}$ (direct interactions), indicating the importance of the mediated couplings. To compare the contribution of the charge transfer states with the one from the higherenergy local excitations, we computed two effective Hamiltonian matrices more: one limiting the outer space to $A^{-} B^{+}$and $A^{+} B^{-}$(Table 5), the other including only the effects of $S_{2}(A) S_{0}(B)$ and $S_{0}(A) S_{2}(B)$ (Table 6 ). As can be seen, the contribution of the charge transfer states $(\sim 0.30 \mathrm{meV})$ predominates on the one from the higher-energy local excitations $(0.03 \mathrm{meV})$.

Table 2: Electronic hamiltonian matrix in the basis of the eight low-lying singlet diabatic states for the dimer model of DPBF shown in Fig. 1. All matrix elements are in units of $\mathrm{meV}$.

\begin{tabular}{c|rrrrrrrr}
\hline$\hat{\mathcal{H}}_{e l}$ & $S_{0}(A) S_{0}(B)$ & $S_{1}(A) S_{0}(B)$ & $S_{0}(A) S_{1}(B)$ & ${ }^{1} T T$ & $A^{-} B^{+}$ & $A^{+} B^{-}$ & $S_{2}(A) S_{0}(B)$ & $S_{0}(A) S_{2}(B)$ \\
\hline$S_{0}(A) S_{0}(B)$ & 0.00 & -13.10 & 12.83 & 0.22 & 18.93 & -18.44 & -115.06 & -115.21 \\
$S_{1}(A) S_{0}(B)$ & -13.10 & 3064.07 & 25.94 & -0.08 & -25.06 & -16.88 & -17.05 & 35.93 \\
$S_{0}(A) S_{1}(B)$ & 12.83 & 25.94 & 3063.99 & 0.08 & -16.92 & -25.16 & -34.71 & 16.89 \\
$1 T T$ & 0.22 & -0.08 & 0.08 & 3656.95 & -24.81 & 25.08 & 0.46 & 0.46 \\
$A^{-} B^{+}$ & 18.93 & -25.06 & -16.92 & -24.81 & 4166.05 & 0.16 & 10.12 & 2.97 \\
$A^{+} B^{-}$ & -18.44 & -16.88 & -25.16 & 25.08 & 0.16 & 4164.24 & -2.99 & -9.61 \\
$S_{2}(A) S_{0}(B)$ & -115.06 & -17.05 & -34.71 & 0.46 & 10.12 & -2.99 & 3766.81 & 43.60 \\
$S_{0}(A) S_{2}(B)$ & -115.21 & 35.93 & 16.89 & 0.46 & 2.97 & -9.61 & 43.60 & 3766.32 \\
\hline
\end{tabular}

Table 3: Coefficients of the diabatic states in the eight low-lying singlet adiabatic states for the dimer model of DPBF shown in Fig. 1. The relative energies $(\mathrm{eV})$ and oscillator strengths of the adiabatic states are also reported.

\begin{tabular}{c|cccccccc|cc}
\hline & $S_{0}(A) S_{0}(B)$ & $S_{1}(A) S_{0}(B)$ & $S_{0}(A) S_{1}(B)$ & ${ }^{1} T T$ & $A^{-} B^{+}$ & $A^{+} B^{-}$ & $S_{2}(A) S_{0}(B)$ & $S_{0}(A) S_{2}(B)$ & Energy & $f\left(S_{0}^{d} \rightarrow S_{n}^{d}\right)$ \\
\hline$S_{0}^{d}$ & -0.999 & -0.004 & 0.004 & 0.000 & 0.005 & -0.004 & -0.030 & -0.030 & 0.000 & - \\
$S_{1}^{d}$ & -0.005 & 0.707 & -0.707 & 0.001 & 0.005 & -0.005 & -0.017 & -0.018 & 3.045 & 0.0000 \\
$S_{2}^{d}$ & 0.000 & -0.704 & -0.705 & 0.000 & -0.027 & -0.027 & -0.057 & 0.058 & 3.091 & 1.3589 \\
$S_{3}^{d}$ & 0.001 & -0.001 & 0.001 & 0.998 & 0.049 & -0.049 & -0.006 & -0.006 & 3.662 & 0.0000 \\
$S_{4}^{d}$ & 0.000 & -0.057 & -0.056 & 0.000 & -0.017 & -0.016 & 0.703 & -0.706 & 3.734 & 0.0053 \\
$S_{5}^{d}$ & 0.043 & -0.018 & 0.017 & -0.011 & 0.023 & -0.022 & -0.707 & -0.705 & 3.824 & 0.7984 \\
$S_{6}^{d}$ & -0.003 & -0.022 & -0.027 & 0.036 & 0.232 & 0.971 & 0.000 & -0.024 & 4.174 & 0.0018 \\
$S_{7}^{d}$ & -0.004 & 0.019 & 0.011 & 0.058 & -0.971 & 0.230 & -0.028 & -0.012 & 4.176 & 0.0016 \\
\hline
\end{tabular}


Table 4: Effective electronic hamiltonian matrix in the basis of the $A\left(S_{1}\right) B\left(S_{0}\right)$, $A\left(S_{0}\right) B\left(S_{1}\right)$ and ${ }^{1} T T$ diabatic states for the dimer model of DPBF shown in Fig. 1. The outer space is spanned by the $A^{-} B^{+}, A^{+} B^{-}, A\left(S_{2}\right) B\left(S_{0}\right)$ and $A\left(S_{0}\right) B\left(S_{2}\right)$ diabatic states. All matrix elements are in units of meV.

\begin{tabular}{c|rrr}
\hline$\hat{\mathcal{H}}_{\text {el }}^{\text {eff }}$ & $S_{1}(A) S_{0}(B)$ & $S_{0}(A) S_{1}(B)$ & ${ }^{1} T T$ \\
\hline$S_{1}(A) S_{0}(B)$ & 3060.82 & 23.25 & -0.43 \\
$S_{0}(A) S_{1}(B)$ & 23.25 & 3060.87 & 0.46 \\
${ }^{1} T T$ & -0.43 & 0.46 & 3654.50 \\
\hline
\end{tabular}

Table 5: Effective electronic hamiltonian matrix in the basis of the $A\left(S_{1}\right) B\left(S_{0}\right)$, $A\left(S_{0}\right) B\left(S_{1}\right)$ and ${ }^{1} T T$ diabatic states for the dimer model of DPBF shown in Fig. 1. The outer space is spanned by the charge transfer $A^{-} B^{+}$and $A^{+} B^{-}$ diabatic states. All matrix elements are in units of meV.

\begin{tabular}{c|rrr}
\hline$\hat{\mathcal{H}}_{\text {el }}^{\text {ef }}$ & $S_{1}(A) S_{0}(B)$ & $S_{0}(A) S_{1}(B)$ & ${ }^{1} T T$ \\
\hline$S_{1}(A) S_{0}(B)$ & 3063.22 & 25.15 & -0.36 \\
$S_{0}(A) S_{1}(B)$ & 25.15 & 3063.13 & 0.38 \\
${ }^{1} T T$ & -0.36 & 0.38 & 3654.51 \\
\hline
\end{tabular}

Table 6: Effective electronic hamiltonian matrix in the basis of the $A\left(S_{1}\right) B\left(S_{0}\right)$, $A\left(S_{0}\right) B\left(S_{1}\right)$ and ${ }^{1} T T$ diabatic states for the dimer model of DPBF shown in Fig. 1. The outer space is spanned by the higher-energy local excitation $A\left(S_{2}\right) B\left(S_{0}\right)$ and $A\left(S_{0}\right) B\left(S_{2}\right)$ diabatic states. All matrix elements are in units of meV.

\begin{tabular}{c|rrr}
\hline$\hat{\mathcal{H}}_{\text {el }}^{\text {ff }}$ & $S_{1}(A) S_{0}(B)$ & $S_{0}(A) S_{1}(B)$ & ${ }^{1} T T$ \\
\hline$S_{1}(A) S_{0}(B)$ & 3061.64 & 24.01 & -0.11 \\
$S_{0}(A) S_{1}(B)$ & 24.01 & 3061.69 & 0.11 \\
${ }^{1} T T$ & -0.11 & 0.11 & 3656.95 \\
\hline
\end{tabular}

\subsection{Exciton coupling in trimers of perylene}

The electronic transitions of a multi-chromophoric system are influenced by the chromophore-to-chromophore interactions, which may cause significant alterations in the spectrum with respect to the isolated chromophores, i.e. band shifts and intensity changes. If the overlaps of the chromophore electron densities are small, so that the chromophore units preserve their individuality in the composite aggregate, the above-mentioned spectral alterations can be explained in many cases in terms of exciton coupling theory [60], i.e. interactions between the localized excited states (excitons) of the individual chromophores. In particular, the lowest adiabatic excited states of the aggregate can be expressed as linear combinations of diabatic states with local excitation character.

As an illustration of the exciton coupling effects, we employed our diabatization procedure to analyze the low-lying adiabatic electronic states of a molec- 
ular trimer, in two different characteristic arrangements. In particular, our model system consists of three interacting molecules of perylene, the parent chromophore of the perylene bisimide class of dyes which exhibit interconversion from H- to J-aggregates [61-63], a property that can be exploited to design new materials for electronic devices, such as solar cells and light-emitting diodes.

In the calculations, we used the AM1 [34] semiempirical Hamiltonian and an active space of 6 electrons in 6 MOs. Moreover, for the determination of floating occupation numbers we employed a gaussian energy width of 0.1 a.u. At this level of theory, using an active space of 2 electrons in 2 orbitals, the first singlet excited state of perylene (point group $D_{2 h}$ ) corresponds to an optically allowed HOMO-to-LUMO one-electron promotion, with $\pi \rightarrow \pi^{*}$ character (state label $\left.1^{1} B_{2 u}\right)$. Furthermore, the associated transition dipole $\vec{\mu}_{01}\left(\mu_{01}^{2}=11.434\right.$ a.u. $)$ lies in the plane of the molecule, along its long axis (see Fig. 3).

We considered two different chromophore arrangements. In the first one (stacked trimer), the three equivalent molecules, each at the $S_{0}$ optimized geometry, are positioned on top of each other with a distance between nearestneighbors of $4.0 \AA$. In the other arrangement (slipped trimer), the molecules, on parallel planes, are slipped along the axis of $\vec{\mu}_{01}$ with a displacement of 8.0 $\AA$ between neighboring molecules (see Fig. 3). While the former arrangement represents a small $\mathrm{H}$-aggregate, the latter can be viewed as a J-aggregate model.

In the determination of the diabatic states, we used four references: the closed-shell Hartree-Fock wavefunction, as a prototype for the ground state diabatic state, hereafter called $A B C$, and three singly excited configurations, each corresponding to the HOMO $\rightarrow$ LUMO transition of a given monomer, as references for diabatic states describing localized excitations, indicated as $A^{*} B C, A B^{*} C$ and $A B C^{*}$.

The computed Hamiltonian matrices in the diabatic basis for both the stacked and slipped trimers are given in Table 7 . Moreover, in Table 8 we report the coefficients of the diabatic states in the adiabatic ones, together with the tran-

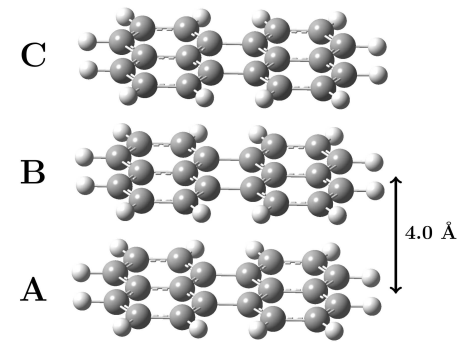

Stacked

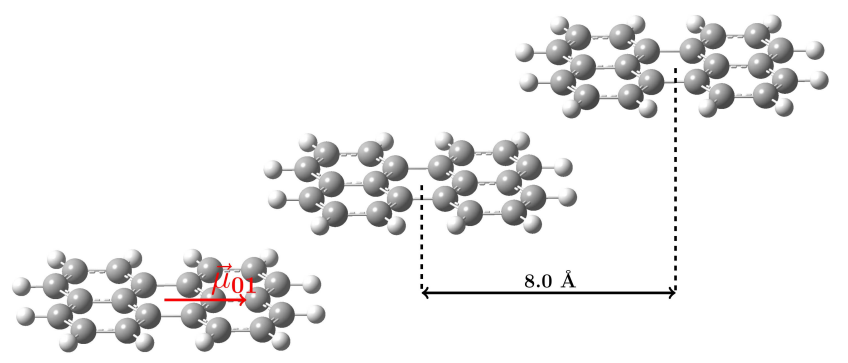

Slipped

Figure 3: Stacked and slipped trimer models of perylene. $A, B$ and $C$ are used to label the monomers. The transition dipole moment for one isolated monomer, $\vec{\mu}_{01}$, is also shown (red arrow). 
Table 7: Electronic hamiltonian matrix in the basis of the four low-lying singlet diabatic states for both the stacked and slipped trimers of perylene shown in Fig. 3. All matrix elements are in units of meV.

\begin{tabular}{c|rrrr}
\hline$\hat{\mathcal{H}}_{e l}$ & $A B C$ & $A^{*} B C$ & $A B^{*} C$ & $A B C^{*}$ \\
\hline Stacked trimer & & & & \\
$A B C$ & 0.00 & 0.00 & 0.00 & 0.00 \\
$A^{*} B C$ & 0.00 & 3377.66 & 203.25 & 50.01 \\
$A B^{*} C$ & 0.00 & 203.25 & 3371.31 & 203.25 \\
$A B C^{*}$ & 0.00 & 50.01 & 203.25 & 3377.66 \\
\hline Slipped trimer & & & & \\
$A B C$ & 0.00 & 3.60 & 0.00 & -3.60 \\
$A^{*} B C$ & 3.60 & 3383.93 & -61.52 & -11.40 \\
$A B^{*} C$ & 0.00 & -61.52 & 3383.09 & -61.52 \\
$A B C^{*}$ & -3.60 & -11.40 & -61.52 & 3383.93 \\
\hline
\end{tabular}

sition dipole moments and the spectral shifts, relative to the isolated monomer, of the adiabatic states.

Table 8: Coefficients of the diabatic states in the four low-lying singlet adiabatic states for both the stacked and slipped trimers of perylene shown in Fig. 3. The relative energies $(\mathrm{eV})$, the spectral shifts $(\mathrm{eV})$ with respect to the $S_{0} \rightarrow S_{1}$ vertical excitation energy of the isolated monomer $(=3.385 \mathrm{eV})$ and transition dipoles squared (a.u.) of the adiabatic states are also reported. The $S_{0} \rightarrow S_{1}$ transition dipole squared of the isolated monomer is 11.434 a.u.

\begin{tabular}{|c|c|c|c|c|c|c|c|}
\hline & $A B C$ & $A^{*} B C$ & $A B^{*} C$ & $A B C^{*}$ & $\begin{array}{c}\text { Energy } \\
(\mathrm{eV})\end{array}$ & $\begin{array}{l}\text { Shift } \\
(\mathrm{eV})\end{array}$ & $\begin{array}{c}\mu_{S_{0} \rightarrow S_{n}}^{2} \\
\text { (a.u.) }\end{array}$ \\
\hline \multicolumn{8}{|c|}{ Stacked trimer } \\
\hline$S_{0}$ & 1.000 & 0.000 & 0.000 & 0.000 & 0.000 & - & - \\
\hline$S_{1}$ & 0.000 & 0.475 & -0.741 & 0.475 & 3.111 & -0.275 & 0.546 \\
\hline$S_{2}$ & 0.000 & 0.707 & 0.000 & -0.707 & 3.328 & -0.058 & 0.000 \\
\hline$S_{3}$ & 0.000 & 0.524 & 0.672 & 0.524 & 3.688 & +0.303 & 30.981 \\
\hline \multicolumn{8}{|c|}{ Slipped trimer } \\
\hline$S_{0}$ & 1.000 & -0.001 & 0.000 & 0.001 & 0.000 & - & - \\
\hline$S_{1}$ & 0.000 & 0.515 & 0.685 & 0.515 & 3.291 & -0.095 & 34.563 \\
\hline$S_{2}$ & 0.002 & 0.707 & 0.000 & -0.707 & 3.395 & +0.010 & 0.000 \\
\hline$S_{3}$ & 0.000 & 0.485 & -0.728 & 0.485 & 3.465 & +0.080 & 0.650 \\
\hline
\end{tabular}

Since in both trimers, molecules $A$ and $C$ are equivalent by symmetry, the weakly interacting, degenerate, diabatic states $A^{*} B C$ and $A B C^{*}$ combine to form two, symmetry adapted, delocalized diabatic states, $\Phi_{+}=\frac{1}{\sqrt{2}}\left(A^{*} B C+\right.$ $\left.A B C^{*}\right)$ and $\Phi_{-}=\frac{1}{\sqrt{2}}\left(A^{*} B C-A B C^{*}\right)$. Moreover, for the same symmetry reasons, the electronic coupling between $\Phi_{-}$and $A B^{*} C$ vanishes and $\Phi_{-}$corresponds to the dark adiabatic excited state $S_{2}$, in both trimers. Conversely, 
$\Phi_{+}$interacts with $A B^{*} C$ and the two diabatic states mix to form the optically allowed adiabatic excited states $S_{1}$ and $S_{3}$. We choose the relative phases of the localized excitations so that the transition dipoles $\left\langle A B C|\hat{\vec{\mu}}| X^{*}\right\rangle$, with $X^{*}=$ $A^{*} B C, A B^{*} C, A B C^{*}$, point in the same direction. Then, if $\left\langle A^{*} B C\left|\hat{\mathcal{H}}_{e l}\right| A B^{*} C\right\rangle$ is positive, as in the case of the stacked trimer, $\Phi_{+}$and $A B^{*} C$ combine with opposite signs in the lower-energy $S_{1}$ state, while they mix with the same phase in the higher-energy $S_{3}$ state. The reverse is true when $\left\langle A^{*} B C\left|\hat{\mathcal{H}}_{e l}\right| A B^{*} C\right\rangle$ is negative (slipped trimer). As a result, while in the stacked trimer the exciton coupling causes a blue-shift of the most intense transition, $S_{0} \rightarrow S_{3}$, by about $0.3 \mathrm{eV}$ relative to the isolated monomer, in the slipped trimer the most intense transition $S_{0} \rightarrow S_{1}$ is red-shifted by about $0.1 \mathrm{eV}$ (see Table 8 ). In both cases, the strongest transition shows an intensity close to that of the separate monomer multiplied by 3 , especially for the slipped trimer.

Assuming that in our models of trimer the molecules are far enough to neglect the short-range exchange interaction deriving from wave function overlap, the results can be qualitatively explained by making use of Kasha's exciton model $[60,64]$, where the couplings between the localized excitations are treated as electrostatic interactions of transition dipole moments (point-dipole approximation). For the two trimer models considered, the application of Kasha's model leads to the diagram shown in Fig. 4. We see that in the stacked trimer the in-phase arrangement of transition dipoles causes repulsion, producing the higher-energy state $S_{3}$, while in the slipped trimer this dipole arrangement gives rise to an electrostatic attraction, leading to the lower-energy state $S_{1}$.

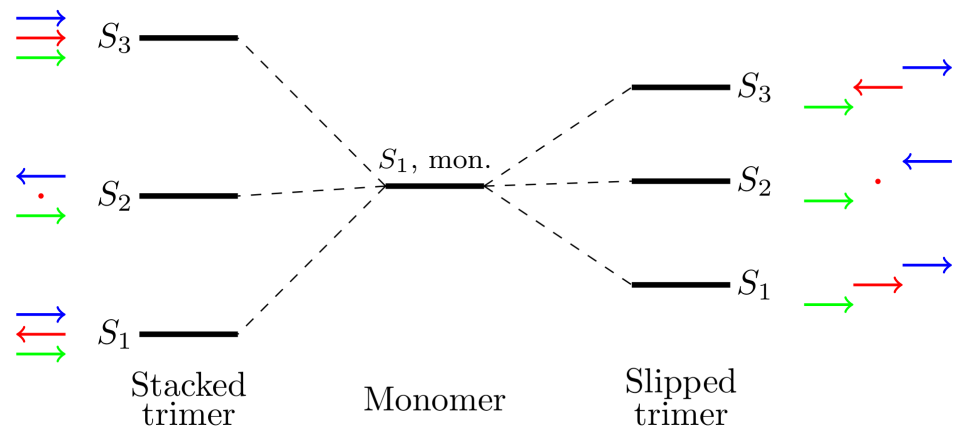

Figure 4: Energy level diagram for the adiabatic states of the two trimer models of Fig. 3. A schematic representation of the transition dipole arrangements is also shown. Each arrow indicates the transition dipole moment of a given monomer. 


\subsection{Cis-trans isomerization and sudden polarization in $1,3,5$-hexatriene}

Although the diabatization method here presented was devised to deal with well separated chromophores, in this section we show that it can also be applied to covalently bound subsystems.

Short polyenes like 1,3-butadiene, 1,3,5-hexatriene and 1,3,5,7-octatetraene have been extensively studied both theoretically $[65,66]$ and experimentally [67-70] with regard to photochemical processes such as cis-trans isomerization and photocyclization. In these photoreactions, the rotation about one or more $\mathrm{C}=\mathrm{C}$ double bonds plays a fundamental role. Moreover, since 1970s polyenes and substituted polyenes have attracted much attention for their tendency to undergo, upon excitation, a strong charge separation in a region around the $90^{\circ}$-twisted geometry. This effect was called "sudden polarization" and has been considered the crucial event in important mechanisms like the vision $[71$, 72], highly stereospecific photocyclizations [73-75] and the photochemistry of provitamin D [76].

Here, we present the calculation of the diabatic states that characterize the three low-lying singlet adiabatic states of 1,3,5-hexatriene (HT) along the twisting around the central double bond. In the attempt to observe the "sudden polarization" effect, we performed this characterization for two different conformers of HT, namely s-trans,s-trans- and s-cis,s-trans-1,3,5-hexatriene (tt-HT and ct-HT, respectively).

In the calculations we used the semiempirical Hamiltonian PM3 [35] with an active space of 2 electrons in 2 MOs $(\mathrm{CAS}(2,2))$. Moreover, for the determination of floating occupations in the SCF procedure we employed an orbital energy width of 0.5 a.u. At this level of theory, at the ground state optimized
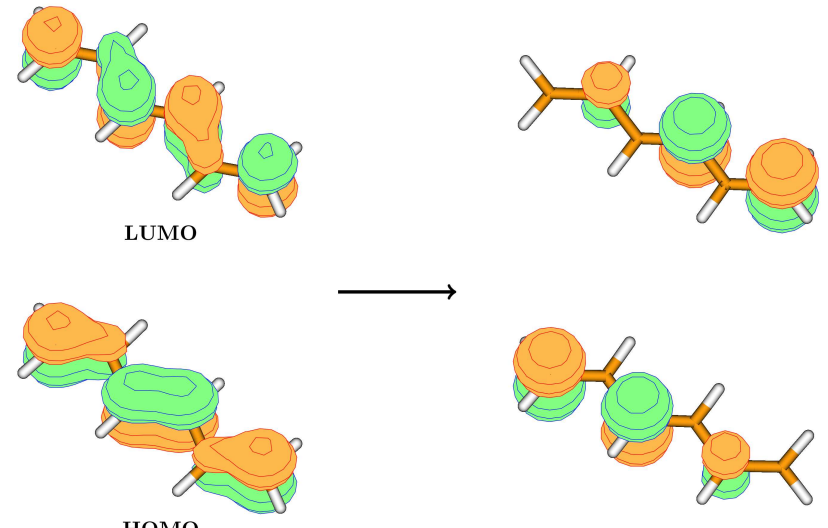

HОМО

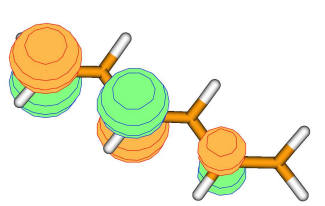

Figure 5: Delocalized (on the left) and localized (on the right) active MOs of tt-HT at the $\mathrm{S}_{0}$ optimized geometry. 


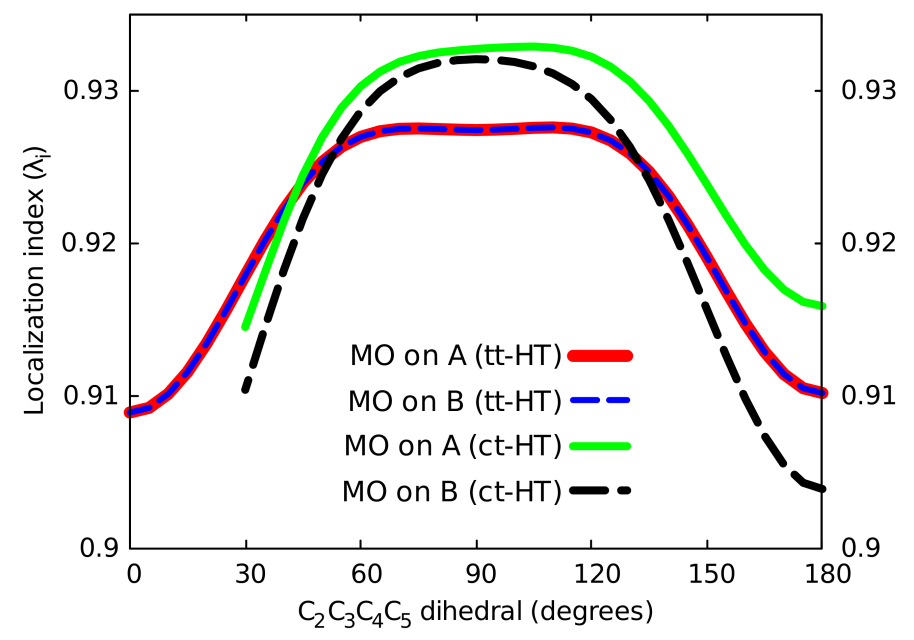

Figure 6: Indices of localizations (eq. 6) of the active MOs for both tt-HT and ct-HT as a function of the $C_{2} C_{3} C_{4} C_{5}$ dihedral angle (called $\theta$ in the main text).

geometry of all-trans-HT (point group $C_{2 h}$ ) the first singlet excited state $S_{1}$ $\left(1^{1} B_{u}\right)$, which corresponds to the lowest bright state, is described as a single HOMO to LUMO transition, while the dark state $S_{2}\left(2^{1} A_{g}\right)$ is mainly a HOMO $\rightarrow$ LUMO double excitation. This state ordering and nature is in agreement with the ab initio and experimental data reported in [77-79].

In the localization of the active MOs, we partitioned the molecule into two allyl fragments, hereafter called $A$ and $B$. As shown in Fig. 5, despite the limited number of MOs employed, our localization method produces quite well localized MOs, with localization indices larger than 0.9 for all values of the twisting angle (Fig. 6).

For the definition of the diabatic states, we used 3 reference states. The first one has a biradical (or covalent) character and corresponds to a configuration where the two localized active MOs are singly occupied. The other two references are ionic configurations where one electron is transferred from one allyl fragment to the other, and viceversa. Since with a CAS $(2,2)$ the CI space consists of only 3 singlet configurations ( 4 determinants), for all the geometries considered the diabatic states obtained are single-configurational wavefunctions corresponding to the reference states.

The potential energy curves of the computed adiabatic and diabatic states of tt-HT, together with the electronic couplings betweeen the diabatic wavefunctions, as a function of the twist angle $\theta\left(=C_{2} C_{3} C_{4} C_{5}\right.$ dihedral angle) are shown in Fig. 7. Moreover, in Fig. 8 we report the coefficients of the diabatic states in the adiabatic wavefunctions along $\theta$.

For all values of $\theta$, the biradical (or covalent) diabatic state $A B$ is slightly above $T_{1}$, with the energy difference given by twice the exchange integral $K_{a b}=$ 


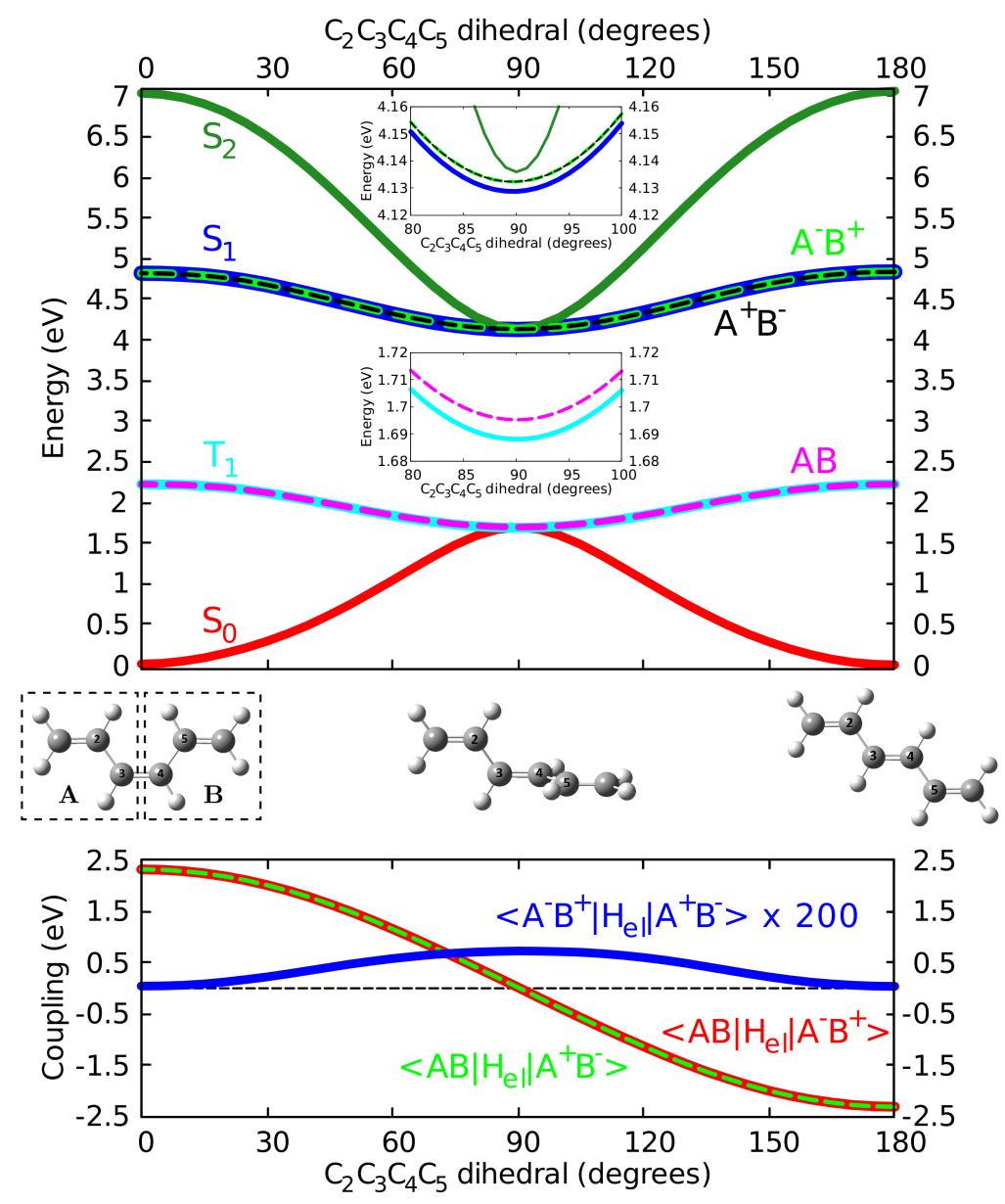

Figure 7: Low-lying adiabatic, diabatic state energies and electronic couplings of tt-HT as a function of the $C_{2} C_{3} C_{4} C_{5}$ dihedral angle (called $\theta$ in the main text). Energies are given relative to the all-trans planar $\left(\theta=180^{\circ}\right)$ ground state optimized conformer. Notation for the diabatic states: $A$ and $B$, allyl fragments; $A B$, biradicaloid (or covalent) state, i.e. $A B=\frac{1}{\sqrt{2}}\left(A^{\uparrow} B^{\downarrow}-A^{\downarrow} B^{\uparrow}\right)$; $A^{-} B^{+}$and $A^{+} B^{-}$, ionic states.

$\left\langle a(1) b(2)\left|\frac{1}{r_{12}}\right| b(1) a(2)\right\rangle$ between the two localized active MOs $a$ and $b$. Moreover, since the two allyl fragments are equivalent by symmetry, the two ionic diabatic states $A^{-} B^{+}$and $A^{+} B^{-}$are degenerate for all values of $\theta$. They combine to form two zwitterionic states, $Z_{1}=\frac{1}{\sqrt{2}}\left(A^{-} B^{+}+A^{+} B^{-}\right)$and $Z_{2}=$ $\frac{1}{\sqrt{2}}\left(A^{-} B^{+}-A^{+} B^{-}\right)$, where the charge distribution is equally divided between the two fragments. Since the interaction between $A^{-} B^{+}$and $A^{+} B^{-}$is very small 


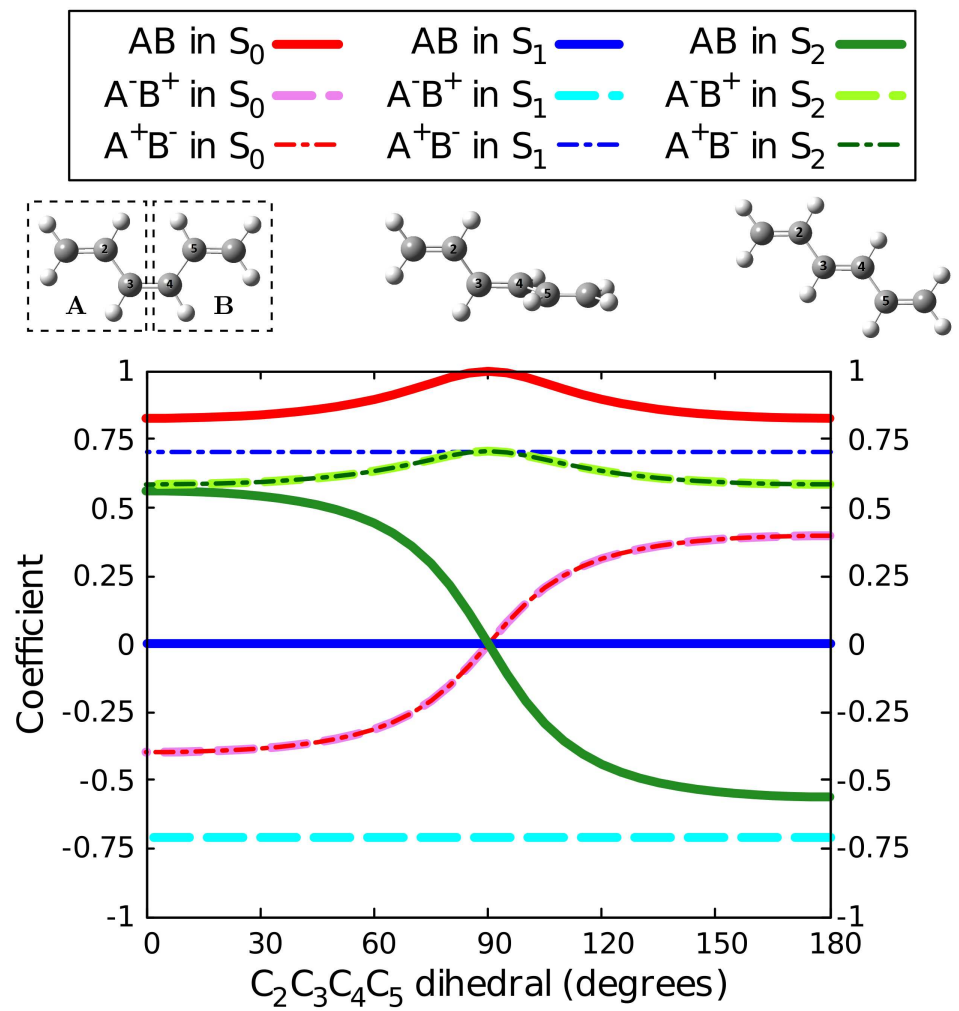

Figure 8: Coefficients of the diabatic states in the low-lying adiabatic ones of tt-HT as a function of the $C_{2} C_{3} C_{4} C_{5}$ dihedral angle (called $\theta$ in the main text). Notation for the diabatic states: $A$ and $B$, allyl fragments; $A B$, biradicaloid (or covalent) state $A B=\frac{1}{\sqrt{2}}\left(A^{\uparrow} B^{\downarrow}-A^{\downarrow} B^{\uparrow}\right) ; A^{-} B^{+}$and $A^{+} B^{-}$, ionic states.

at all $\theta$ values, $\left\langle A^{-} B^{+}\left|\hat{\mathcal{H}}_{e l}\right| A^{+} B^{-}\right\rangle=K_{a b} \approx 0, Z_{1}$ and $Z_{2}$ are approximately degenerate. However, since $\left\langle A B\left|\hat{\mathcal{H}}_{e l}\right| A^{-} B^{+}\right\rangle=\left\langle A B\left|\hat{\mathcal{H}}_{e l}\right| A^{+} B^{-}\right\rangle, Z_{2}$ does not interact with the biradical state $A B$ and contributes only to $S_{1}$, whereas at planar geometries the coupling between $Z_{1}$ and $A B$ is large and the two diabatic states combine to form $S_{0}$ and $S_{2}$. In the twisted conformation $\left(\theta=90^{\circ}\right)$, both $\left\langle A B\left|\hat{\mathcal{H}}_{e l}\right| Z_{1}\right\rangle$ and $\left\langle A^{-} B^{+}\left|\hat{\mathcal{H}}_{e l}\right| A^{+} B^{-}\right\rangle$are very small and it turns out that $S_{0} \simeq A B, S_{2}=Z_{1}$ and $S_{1} \simeq Z_{2}$ (Fig. 8). Therefore, while the purely zwitterionic $S_{1}$ state shows a weak dependence on $\theta, S_{0}$ and $S_{2}$ undergo a significant change in their nature, which results in a faster variation along the twist coordinate.

If we perform a similar study on ct-HT, we obtain potential energy curves (Fig. 9) very similar to the ones of tt-HT, at least for $30^{\circ} \leq \theta \leq 180^{\circ}$ (in this case we have excluded the geometries with $0^{\circ} \leq \theta<30^{\circ}$, where two hydrogens atoms come very close to each other). However, if we look at the 


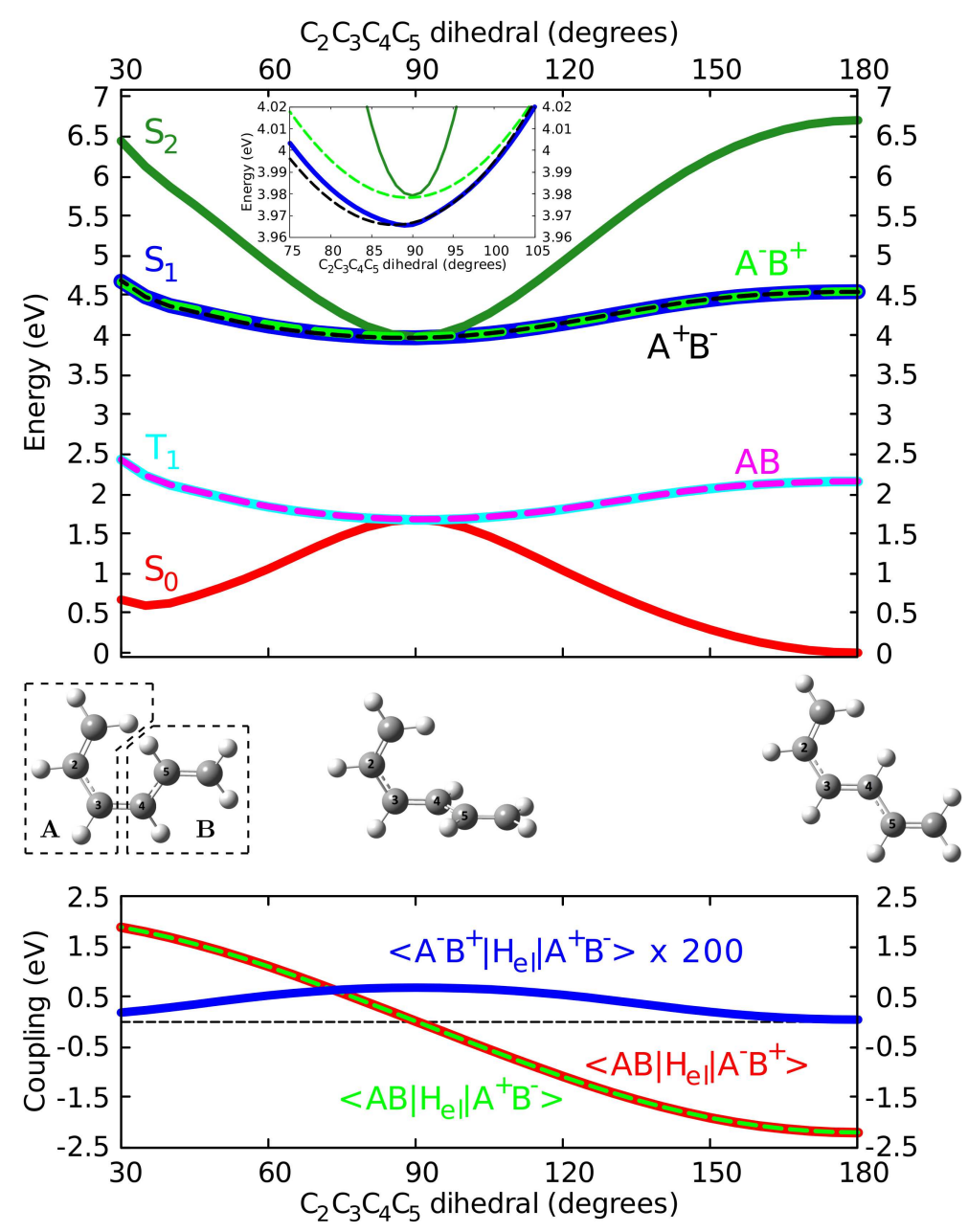

Figure 9: Low-lying adiabatic, diabatic state energies and electronic couplings of ct-HT as a function of the $C_{2} C_{3} C_{4} C_{5}$ dihedral angle (called $\theta$ in the main text). Energies are given relative to the s,cis-s, trans planar $\left(\theta=180^{\circ}\right)$ ground state optimized conformer. Notation for the diabatic states: $A$ and $B$, allyl fragments; $A B$, biradicaloid (or covalent) state, i.e. $A B=\frac{1}{\sqrt{2}}\left(A^{\uparrow} B^{\downarrow}-A^{\downarrow} B^{\uparrow}\right)$; $A^{-} B^{+}$and $A^{+} B^{-}$, ionic states.

coefficients of the diabatic states in the adiabatic ones as a function of $\theta$ (Fig. 10), we observe a significant polarization of the two excited states $S_{1}$ and $S_{2}$ in a narrow range around the $90^{\circ}$-twisted geometry. According to our calculations, in the explanation of the "sudden polarization" effect the electronic couplings $\left\langle A B\left|\hat{\mathcal{H}}_{e l}\right| A^{-} B^{+}\right\rangle$and $\left\langle A B\left|\hat{\mathcal{H}}_{e l}\right| A^{+} B^{-}\right\rangle$, together with the degeneracy 


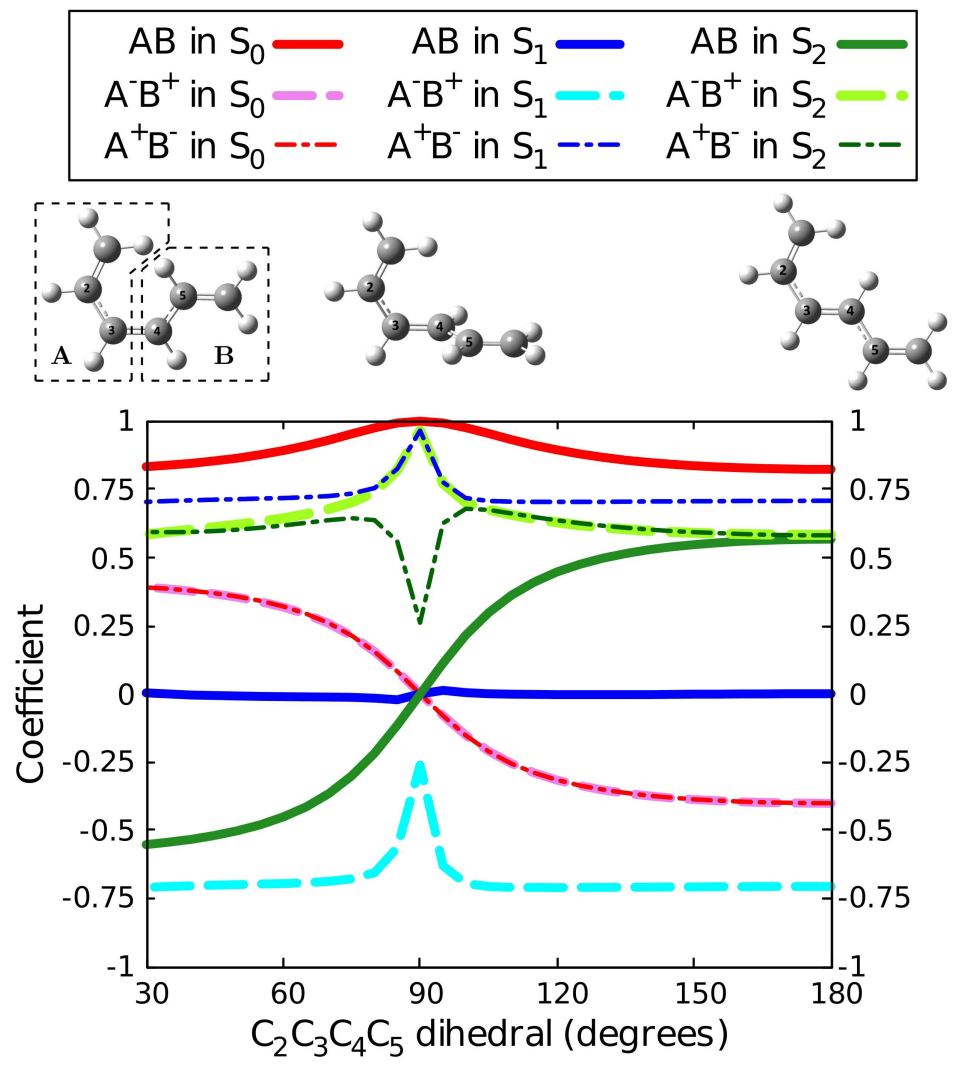

Figure 10: Coefficients of the diabatic states in the low-lying adiabatic ones of ct-HT as a function of the $C_{2} C_{3} C_{4} C_{5}$ dihedral angle (called $\theta$ in the main text). Notation for the diabatic states: $A$ and $B$, allyl fragments; $A B$, biradicaloid (or covalent) state $A B=\frac{1}{\sqrt{2}}\left(A^{\uparrow} B^{\downarrow}-A^{\downarrow} B^{\uparrow}\right) ; A^{-} B^{+}$and $A^{+} B^{-}$, ionic states.

breaking between $A^{-} B^{+}$and $A^{+} B^{-}$around $\theta=90^{\circ}$, play the fundamental role. At twist angles away from $90^{\circ}$, where $\left\langle A B\left|\hat{\mathcal{H}}_{e l}\right| A^{-} B^{+}\right\rangle \simeq\left\langle A B\left|\hat{\mathcal{H}}_{e l}\right| A^{+} B^{-}\right\rangle>>$ $\left\langle A^{-} B^{+}\left|\hat{\mathcal{H}}_{e l}\right| A^{+} B^{-}\right\rangle,\left\langle A B\left|\hat{\mathcal{H}}_{e l}\right| A^{-} B^{+}\right\rangle$and $\left\langle A B\left|\hat{\mathcal{H}}_{e l}\right| A^{+} B^{-}\right\rangle$predominate in the determination of the adiabatic states, imposing an almost symmetrical charge destribution. For $\theta \simeq 90^{\circ},\left\langle A B\left|\hat{\mathcal{H}}_{e l}\right| A^{-} B^{+}\right\rangle$and $\left\langle A B\left|\hat{\mathcal{H}}_{e l}\right| A^{+} B^{-}\right\rangle$vanish and $\left\langle A^{-} B^{+}\left|\hat{\mathcal{H}}_{e l}\right| A^{+} B^{-}\right\rangle$is too small, i.e. much smaller than the energy difference between $A^{-} B^{+}$and $A^{+} B^{-}$, to prevent the charge separation in the excited states. In agreement with previous calculations [74,75], in the $S_{1}$ state the negative charge prefers the s, trans subsystem $(B)$, while in $S_{2}$ the negative charge suddenly flows into the s,cis fragment $(A)$ of the molecule (see Fig. 10). This sudden charge separation is confirmed by the Mulliken population analysis of the adiabatic wavefunctions (see Fig. S1). 


\section{Conclusions}

In this paper we have presented a diabatization method of general applicability. The method is based on the localization of MOs on user specified groups of atoms, and on an orthogonal transformation of the adiabatic basis, defined by requiring maximum overlap with a set of diabatic references, built on the localized MOs.

We have shown how to apply the procedure to compute the low-lying diabatic states and electronic couplings involved in singlet fission and exciton coupling for systems consisting of well separated chromophores. Moreover, we have successfully tested the method on an example of covalently bound subsystems, namely the cis-trans isomerization in hexatriene. In this test application, we have shown that the method is able to describe processes where the system evolves from strong interaction regions to geometries where the electronic couplings are weak (or vice versa). This feature is particularly important for the application of the algorithm to analyze adiabatic or time-dependent electronic wavefunctions, obtained in excited state dynamics simulations.

Although we have presented the diabatization procedure as implemented in the framework of semiempirical FOMO-CI $[5,10]$, the same transformation can be applied to ab initio wavefunctions, computed for instance with the stateaverage CASSCF or the ab initio FOMO-CI $[27,28]$ method.

\section{Acknowledgment}

This work was performed thanks to grant PRA_2018_36 of the University of Pisa.

\section{References}

[1] M. Persico, in R. Schleyer, N. L. Allinger, T. Clark, J. Gasteiger, P. A. Kollman, H. F. Schaefer III, P. R. Schreiner, eds., The Encyclopedia of Computational Chemistry, vol. 2, Wiley, Chichester, 1998, 852-860.

[2] L. S. Cederbaum, in W. Domcke, D. R. Yarkony, H. Köppel, eds., Conical Intersections. Electronic Structure, Dynamics 83 Spectroscopy, World Scientific, Singapore, 2004, 3-40.

[3] H. Köppel, in W. Domcke, D. R. Yarkony, H. Köppel, eds., Conical Intersections. Electronic Structure, Dynamics \& Spectroscopy, World Scientific, Singapore, 2004, 175-204.

[4] C. Curutchet, B. Mennucci, Chem. Rev. 2017, 117, 294-343.

[5] M. Persico, G. Granucci, Theor. Chem. Acc. 2014, 133, 1526/1-28. 
[6] M. Persico, G. Granucci, Photochemistry: A Modern Theoretical Perspective., Theoretical Chemistry and Computational Modelling Series, Springer, Cham, 2018.

[7] J. Vura-Weis, M. D. Newton, M. R. Wasielewski, J. E. Subotnik, J. Phys. Chem. C 2010, 114, 20449-20460.

[8] R. J. Cave, M. D. Newton, J. Phys. Chem. A 2014, 118, 7221-7234.

[9] M. Wibowo, M. Persico, G. Granucci, Phys. Chem. Chem. Phys. 2019, 21, 692-701.

[10] G. Granucci, M. Persico, A. Toniolo, J. Chem. Phys. 2001, 114, 1060810615 .

[11] H. Köppel, B. Schubert, Mol. Phys. 2006, 104, 1069-1079.

[12] F. Plasser, G. Granucci, J. Pittner, M. Barbatti, M. Persico, H. Lischka, J. Chem. Phys. 2012, 137, 22A514/1-13.

[13] X. Zhu, D. R. Yarkony, J. Chem. Phys. 2016, $144,044104$.

[14] G. W. Richings, G. A. Worth, Chem. Phys. Letters 2017, 683, 606-612.

[15] L. Joubert-Doriol, B. Lasorne, D. Lauvergnat, H.-D. Meyer, F. Gatti, J. Chem. Phys. 2014, 140, 044301/1-15.

[16] I. Burghardt, J. T. Hynes, J. Phys. Chem. A 2006, 110, 11411-11423.

[17] H. Tamura, R. Martinazzo, M. Ruckenbauer, I. Burghardt, J. Chem. Phys. 2012, 137, 22A540/1-8.

[18] R. Cimiraglia, J. P. Malrieu, M. Persico, F. Spiegelmann, J. Phys. B 1985, 18, 3073-3084.

[19] P. Cattaneo, M. Persico, Chem. Phys. Letters 1996, 262, 747-750.

[20] A. Troisi, G. Orlandi, J. Chem. Phys. 2003, 118, 5356-5363.

[21] J. E. Subotnik, R. J. Cave, R. P. Steele, S. N., J. Chem. Phys. 2009, 130, 234102/1-14.

[22] A. F. Morrison, Z.-Q. You, J. M. Herbert, J. Chem. Theory Comput. 2014, $10,5366-5376$.

[23] A. A. Voityuk, J. Chem. Phys. 2014, 140, 244117/1-7.

[24] A. Grofe, Z. Qu, D. G. Truhlar, H. Li, J. Gao, J. Chem. Theory Comput. 2017, 13, 1176-1187.

[25] M. Wibowo, R. Broer, R. W. A. Havenith, Comput. Theoret. Chem. 2017, $1116,190-194$. 
[26] M. F. S. J. Menger, F. Plasser, B. Mennucci, L. González, J. Chem. Theory Comput. 2018, 14, 6139-6148.

[27] A. Toniolo, M. Persico, D. Pitea, J. Chem. Phys. 2000, 112, 2790-2797.

[28] D. Hollas, L. Šištík, E. G. Hohenstein, T. J. Martínez, P. Slavíček, J. Chem. Theory Comput. 2018, 14, 339-350.

[29] P. de Silva, M. Giebultowski, J. Korchowiec, Phys. Chem. Chem. Phys. 2012, 14, 546-552.

[30] C. Zhang, S. Li, J. Chem. Phys. 2014, 141, 244106/1-8.

[31] I. Mayer, Chem. Phys. Letters 2007, 437, 284-286.

[32] P.-O. Löwdin, Adv. Quant. Chem. 1970, 5, 185-199.

[33] M. J. Dewar, W. Thiel, J. Am. Chem. Soc. 1977, 99, 4899-4907.

[34] M. J. S. Dewar, E. G. Zoebisch, E. F. Healy, J. J. P. Stewart, J. Am. Chem. Soc. 1985, 107, 3902-3909.

[35] J. J. P. Stewart, J. Comput. Chem. 1989, 10, 221-264.

[36] J. J. P. Stewart, J. Molec. Model. 2007, 13, 1173-1213.

[37] G. Granucci, A. Toniolo, Chem. Phys. Lett. 2000, 325, 79-85.

[38] T. Cusati, E. Martínez-Núñez, F. Martini, M. Persico, S. Vázquez, J. Phys. Chem. A 2012, 116, 98-110.

[39] M. B. Smith, J. Michl, Chem. Rev. 2010, 110, 6891-6936.

[40] M. B. Smith, J. Michl, Ann. Rev. Phys. Chem. 2013, 64, 361-386.

[41] A. Japahuge, T. Zeng, ChemPlusChem 2018, 83, 146-182.

[42] D. Casanova, Chem. Rev. 2018, 118, 7164-7207.

[43] A. Rao, M. W. B. Wilson, J. Hodgkiss, S. Albert-Seifried, H. Bassler, H. R. Friend, J. Am. Chem. Soc. 2010, 132, 12698-12703.

[44] M. W. B. Wilson, A. Rao, J. Clark, R. S. S. Kumar, D. Brida, G. Cerullo, R. H. Friend, J. Am. Chem. Soc. 2011, 133, 11830-11833.

[45] M. C. Hanna, A. J. Nozik, J. Appl. Phys. 2006, 100, 1-8.

[46] W. Shockley, H. J. Queisser, J. Appl. Phys. 1961, 32, 510-519.

[47] E. A. Buchanan, Z. Havlas, J. Michl, Adv. Quant. Chem. 2017, 75, 175227.

[48] X. Feng, A. B. Kolomeisky, A. I. Krylov, J. Phys. Chem. C 2014, 118, 19608-19617. 
[49] D. Accomasso, G. Granucci, R. Havenith, M. Persico, Chem. Phys. 2018, $515,635-642$.

[50] N. Monahan, X.-Y. Zhu, Ann. Rev. Phys. Chem. 2015, 66, 601-618.

[51] I. Paci, J. C. Johnson, X. Chen, G. Rana, D. Popovic, D. E. David, A. J. Nozik, M. A. Ratner, J. Michl, J. Am. Chem. Soc. 2006, 128, 16546-16553.

[52] J. C. Johnson, J. Michl, Top. Curr. Chem. (Z) 2017, 375, 80/1-29.

[53] J. L. Ryerson, J. N. Schrauben, A. J. Ferguson, S. C. Sahoo, P. Naumov, Z. Havlas, J. Michl, A. J. Nozik, J. C. Johnson, J. Phys. Chem. C 2014, 118, 12121-12132.

[54] J. C. Johnson, A. J. Nozik, J. Michl, J. Am. Chem. Soc. 2010, 132, 1630216303.

[55] Z. Havlas, J. Michl, Israel J. Chem. 2016, 56, 96-106.

[56] E. A. Buchanan, J. Michl, J. Am. Chem. Soc. 2017, 139, 15572-15575.

[57] A. F. Schwerin, J. C. Johnson, M. B. Smith, P. Sreearunothai, D. Popović, J. Černý, Z. Havias, I. Paci, A. Akdag, M. K. MacLeod, X. Chen, D. E. David, M. A. Ratner, J. R. Miller, A. J. Nozik, J. Michl, J. Phys. Chem. A 2010, 114, 1457-1473.

[58] E. C. Greyson, B. R. Stepp, X. Chen, A. F. Schwerin, I. Paci, M. B. Smith, A. Akdag, J. C. Johnson, A. J. Nozik, J. Michl, M. A. Ratner, J. Phys. Chem. B 2010, 114, 14223-14232.

[59] W. Herkstroeter, P. Merkel, J. Photochem. 1981, 16, 331-341.

[60] M. Kasha, H. R. Rawls, M. A. El-Bayoumi, Pure Appl. Chem. 1965, 11, 371-392.

[61] S. Ghosh, X. Li, V. Stepanenko, F. Wurthner, Chem. Eur. J. 2008, 14, 11343-11357.

[62] S. Yagai, T. Seki, T. Karatsu, A. Kitamura, F. Wurthner, Angew. Chem. Int. Ed. 2008, 47, 3367-3371.

[63] A. Sarbu, L. Biniek, J. Guenet, P. J. Mesini, M. Brinkmann, J. Mater. Chem. C 2015, 3, 1235-1242.

[64] M. Kasha, Radiat. Res. 1963, 20, 55-70.

[65] M. Olivucci, F. Bernardi, P. Celani, I. Ragazos, M. A. Robb, J. Am. Chem. Soc. 1994, 116, 1077-1085.

[66] M. Garavelli, P. Celani, F. Bernardi, M. A. Robb, M. Olivucci, J. Am. Chem. Soc. 1997, 119, 11487-11494. 
[67] C. C. Hayden, D. W. Chandler, J. Phys. Chem. 1995, 99, 7897-7903.

[68] O. Kaoru, N. Yukito, S. Kenichi, T. Keisuke, H. Noboru, Y. Keitaro, Chem. Phys. Lett. 1996, 256, 629-634.

[69] W. Fuss, T. Schikarski, W. E. Schmid, S. A. Trushin, P. Hering, K. L. Kompa, J. Chem. Phys. 1997, 106, 2205-2211.

[70] W. G. Dauben, M. S. Kellogg, J. Am. Chem. Soc. 1972, 94, 8951-8953.

[71] L. Salem, Acc. Chem. Res. 1979, 12, 87-92.

[72] F. Blomgren, S. Larsson, Int. J. Quant. Chem. 2002, 90, 1536-1546.

[73] W. G. Dauben, J. S. Ritscher, J. Am. Chem. Soc. 1970, 92, 2925-2926.

[74] V. Bonačić-Koutecký, P. Bruckmann, P. Hiberty, J. Koutecký, C. Leforestier, L. Salem, Angew. Chem. Int. Ed. 1975, 14, 575-576.

[75] V. Bonačić-Koutecký, J. Am. Chem. Soc. 1978, 100, 396-402.

[76] H. J. C. Jacobs, F. Boomsma, E. Havinga, A. van der Gen, Recl. Trav. Chim. Pays-Bas 1977, 96, 113-117.

[77] L. Serrano-Andrés, M. Merchán, I. Nebot-Gil, R. Lindh, B. O. Roos, J. Chem. Phys. 1993, 98, 3151-3162.

[78] O. A. Mosher, A. Kuppermann, W. M. Flicker, Chem. Phys. Lett. 1977, 45, 492-497.

[79] T. Fujii, A. Kamata, M. Shimizu, Y. Adachi, S. Maeda, Chem. Phys. Lett. 1985, 115, 369-372.

DOI: $10.1002 /$ cptc. 201900056

Supporting Information available online. 